\title{
Molecular tests to detect human papillomavirus infection in patients with cervical dysplasia and invasive cervical cancer in Saudi Arabia
}

This article was published in the following Dove Press journal:

Pathology and Laboratory Medicine International

27 July 2011

Number of times this article has been viewed

\author{
Khalid H Sait ${ }^{\prime}$ \\ Faten S Gazzaz ${ }^{2}$ \\ 'Obstetrics and Gynecology \\ Department, ${ }^{2}$ Medical Virology \\ Department, Faculty of Medicine, King \\ Abdulaziz University, Jeddah, Saudi \\ Arabia
}

Purpose: The aim of this study was to determine the actual human papillomavirus (HPV) subtype that presents in cervical dysplasia and invasive carcinoma in the Saudi population, and the feasibility of using Hybrid Capture 2 technique (HC2) on biopsy specimens to detect certain HPV subtypes.

Patients and methods: A prospective study was conducted from March 2007 to December 2008. The subjects studied were women with a mean age of 48.18 years, who attended the hospital for cervical biopsy due to the suspected diagnosis of cervical dysplasia or an invasive disease, based on previous suspicious Pap smear. HPV DNA hybridization by HC2 was performed on the cervical biopsies of these patients, to detect HPV infection.

Results: During the period of this study, 45 patients had cervical biopsies taken for HPV testing. Seven patients had a negative $\mathrm{HC} 2$ result and were found to have no cervical dysplasia on the final pathology review. Seventeen cases with cervical dysplasia and 21 patients with invasive disease were presented; the mean age was 48 years. HC2 testing for HPV were found to be positive in patients with cervical dysplasia, invasive carcinoma, and all in 5 (29.4\%), 13 (61.9\%) and 18 (47.4\%), respectively. The sensitivity of the test is $47 \%$ and specificity is $100 \%$.

Conclusion: The use of molecular detection of HPV DNA by HC2 in biopsy is feasible and effective. These results confirm the finding that HPV contributes to the etiology of cervical cancer in Muslim society.

Keywords: HPV, subtyping, cervical neoplasia

\section{Introduction}

Cervical cancer is the second cause of cancer death in women globally and the number one cause of cancer death in women in many developing countries. ${ }^{1,2}$ The occurrence of cervical carcinoma is low in Saudi women. According to the Saudi National Cancer Registry 2005 Report, cancer of the cervix is the eleventh most frequent cancer in women, and the eighth between the ages of 15 and 44 years old. Current estimates in Saudi Arabia indicate that every year, 152 women are diagnosed with cervical cancer and 55 die from the disease. Experts believe that essentially all cases of cervical cancer are caused by infection with oncogenic types of human papillomavirus (HPV). ${ }^{4-6}$ Highly safe, immunogenic, and effective vaccines have recently been developed that can prevent infection with certain types of HPV (types 16 and 18), which are responsible for more than $70 \%$ of cervical cancer. ${ }^{7,8}$ These vaccines have now been licensed by the National Control authorities in more than 80 countries. In addition, expert committees in many countries have issued recommendations for the use of the vaccines. Irrespective of the global geographic region, the most common HPV types found in association 
with anogenital cancers are types 16 and 18, referred to as high-risk (HR) groups. ${ }^{7}$ Cancer of the cervix is by far the most common genital cancer and $70 \%$ of cervical cancers are caused by types 16 and $18 .{ }^{8}$ Other genital HPV types do not cause cancer, but can cause diseases like genital warts, which are referred to as a low-risk (LR) group. ${ }^{9,10}$ Relatively few HPV prevalence studies are available from Middle Eastern countries. However, it is likely that the prevalence and the incidence of HPV infection will vary between countries in the region, because of different sexual behaviors and societal norms. Overall, the prevalence of HPV in Saudi Arabia is likely to be lower than other countries in the world; according to Gazzaz, HPV prevalence is as low as $5 \% .{ }^{11}$

While waiting for larger prevalence studies on HPV in our general population, the authors conducted this pilot study on the prevalence of HPV in cervical dysplasia and invasive carcinoma in the Saudi population using the Hybrid Capture 2 technique (HC2) in biopsy specimens.

\section{Materials and methods}

This pilot study was done on cervical biopsies performed on patients with cervical dysplasia and invasive disease, to test for HPV in the Saudi population.

\section{Collection of cervical biopsies for HPV DNA testing}

Freshly collected cervical biopsies $2-5 \mathrm{~mm}$ in cross section were collected immediately into a $1.0 \mathrm{~mL}$ of Digene liquid collection medium (specific for the transport of HPV DNA) via a sample of cells taken from the cervix and transported to the virology laboratory. In the absence of cervical mass, biopsy was done under colposcopy guide on patients referred with an abnormal Pap smear, and requiring colposcopy according to the Bethesda system. Two biopsies were obtained, sent as routine histopathology, and placed in formalin. The other biopsy specimen was stored frozen at $-20^{\circ} \mathrm{C}$ until processed for HPV testing.

\section{Methodology}

After DNA extraction from the tissue, the HC2 an HPV DNA test, was performed according to the specifications of the manufacturer (Digene Corporation, Gaithersburg, MD), using the HPV DNA Test (Two-Probe Method). Each specimen was tested twice; once with LR Probe cocktail (LR types $6,11,42,43$, and 44) and then with HR Probe cocktail (HR types $16,18,31,33,35,39,45,51,52,56,58,59$, and 68 ). These tests determined whether the specimens were positive or negative for HR and LR HPV types.
According to the principle of the HC2 HPV DNA test procedure, the specimens containing the target DNA hybridize with a specific HPV RNA probe. The resultant RNA:DNA hybrids are captured onto the surface of a Microplate well coated with antibodies specific for RNA:DNA hybrids. Immobilized hybrids were then reacted with alkaline phosphatase conjugated antibodies specific for the RNA:DNA hybrids, and detected with a chemiluminescent substrate. Several alkaline phosphatase molecules are conjugated to each antibody. Multiple conjugated antibodies bind to each captured hybrid resulting in substantial signal amplification. As the substrate is cleared by the bound alkaline phosphatase, light emitted is measured as relative light units (RLUs) on a luminometer. The intensity of the light emitted denotes the presence or absence of target DNA in the specimen.

The interpretations of the test result were performed according to the manufacturer's instructions. Briefly, RLU measurement is equal to or is greater than the cut-off value indicated by the presence of HPV DNA sequences in the specimen. When the RLU measurement is less than the cutoff value, this indicates the absence of the specific HPV DNA sequences tested, or HPV DNA levels below the detection limit of the assay. These measurements were performed by the Digene hybrid capture system (DHCS v.2) software and DML 2000 instrument.

\section{Assay set-up}

The specimens and all required HC2 HPV DNA test kit reagents were allowed to reach $20^{\circ} \mathrm{C}-25^{\circ} \mathrm{C}$ (room temperature) for 15 to 30 minutes. Microplate heater I was pre-warmed to $65^{\circ} \mathrm{C} \pm 2{ }^{\circ} \mathrm{C}$ for at least 60 minutes from a cold start. The water bath was confirmed to be at $65^{\circ} \mathrm{C}$, and the water level had to be high enough to immerse the entire volume in the specimen tubes. Specimen lists were prepared and plate layouts assayed in Digene hybrid capture system (DHCS v.2) software. Calibrators, quality controls, and specimens to be tested were placed in a test tube rack in the same order in which they were tested. Run was done for Negative Calibrator (NC), HR HPV Calibrator (H-RC), or LR HPV Calibrator (L-RC), Low-Risk Quality Control (QC1-LR), and High-Risk Quality Control (QC2-HR), plus specimens in an 8-microplate well column configuration. NC, $\mathrm{H}-\mathrm{RC}$, or L-RC were tested in triplicate, and QC1-LR and QC2-HR were tested singly with H-RC HPV or L-RC Probe Mix. The study was conducted at King Abdulaziz University Hospital, Jeddah, Kingdom of Saudi Arabia between March 2007 and December 2008. The study was approved by the Institutional Review Board. 


\section{Results}

During the period of the study, 45 patients had cervical biopsies taken for HPV testing. Seven patients had negative LR and HR HPV using HC2 and were found to have no cervical dysplasia on the final pathology review; 17 cases with cervical dysplasia and 21 patients with invasive carcinoma were present with a mean age of 48 years (range 27-78). Each patient had one or two biopsies taken for testing. The results obtained by $\mathrm{HC} 2$ were positive in cervical dysplasia, invasive carcinoma, and overall in five (29.4\%), 13 (61.9\%), and 18 (47.4\%), respectively (Table 1). The majority of the patients with cervical dysplasia were low grade (15 patients; 88.2\%) and the remaining two patients high grade. Five tested positive with four HR HPV (three low grade dysplasia and one high grade) and one LR HPV (low grade dysplasia). However, one of three patients with low grade dysplasia and positive for HR HPV was found 6 months later to have high grade dysplasia. Among cases of invasive carcinoma with positive HR HPV testing, eleven were squamous cell carcinoma and two were adenocarcinoma. The sensitivity of the LR and HR HPV using $\mathrm{HC} 2$ is $47 \%$ and specificity is $100 \%$.

RLU values in relation to different histopathology diagnoses in positive HPV DNA cervical biopsies are demonstrated in Figure 1. There was a highly significant difference in the RLUs between those women who were reported as having HR HPV infection, and those who had negative HPV DNA results. By comparing the RLU values of positive specimens, we found that the signal generated from positive specimens was as high as $1,969,854$ RLUs, in patients with invasive disease, but as low as 2069 RLUs in patient with cervical dysplasia especially of low grade.

\section{Discussion}

In Saudi Arabia the incident rate of cervical cancer is one of the lowest in the world at 1.3 per 100,000 women, comprising $2.6 \%$ of diagnosed cancer cases. ${ }^{3}$ Pap smear screening, which identifies cytological abnormalities of the cervical transformation zone, has helped reduced cervical cancer incidence and mortality rates by $70 \%$ in developed countries. ${ }^{12}$ An intensive

Table I HPV testing in cervical dysplasia and invasive disease

\begin{tabular}{lllll}
\hline \multirow{2}{*}{ Results } & \multicolumn{2}{l}{ Cervical dysplasia } & Invasive carcinoma & Total \\
\cline { 2 - 3 } & LGSIL & HGSIL & & \\
\hline Positive & $4(37 \%)^{*}$ & I (50\%) & I3 (62\%) & $18(47 \%)$ \\
Negative & II (63\%) & I (50\%) & $8(38 \%)$ & $20(53 \%)$ \\
Total & I5 & 2 & 2 I & 38 \\
\hline
\end{tabular}

Note: *One LGSIL was found to be low risk HPV.

Abbreviations: HGSIL, high grade squamous intraepithelial lesion; LGSIL, low grade squamous intraepithelial lesion. screening program for cervical cancer in Saudi Arabia is not well established, and despite the availability of the Pap smear test among different health sectors, it has been noted that the number of cases of cervical cancer has been increasing over the past two decades. An opportunistic screening was carried out by health care workers. When an abnormality was detected in a Pap smear, the patient was referred for colposcopy-directed biopsy, or a direct biopsy for obvious invasive carcinoma. Conventional cytology exists and there is limited hospital-based data on abnormalities, but some limited, population-based information showed an overall reported abnormality for Pap smear ranges from 2\%-5\%. ${ }^{13,14}$

Because HPV cannot be cultured reliably in a laboratory setting, HPV diagnostics rely on molecular technologies that detect HPV DNA in cervical/vaginal samples present in each specimen. Several diagnostic technologies are also able to estimate a specimen's viral load, which approximates the average number of viral genomes in the cervical cells sampled. Whether such semi-quantitative data yield clinically relevant information has not yet been determined.

Signal-amplified techniques for detecting HPV, include hybrid capture and branched DNA approaches. The most widely used technique is hybrid capture (HC) technology.

HC technology detects nucleic acid targets directly, using signal amplification to provide sensitivity comparable to target amplification methods. Digene has developed two products for the detection of HPV: the first-generation Hybrid Capture Tube (HCT) test and the more recent HC2 assay. Both assays detect HR HPV types. The HCT test detects the following HR types: 16, 18, 31, 33, 35, 45, 51, 52, and 56 . HCT was granted US Food and Drug Administration (FDA) approval in May 1995. In March 1999, the US FDA approved Digene's second-generation HPV detection kit (HC2). In our center, HC2 was used to test for LR and HR HPV, and was found to have $100 \%$ specificity.

Certain social and moral standards could make Muslim women less susceptible to HPV infection. ${ }^{13-16}$ Cervical cancer is both preventable and curable. In Saudi Arabia, most cases are presented at advanced stages ${ }^{17,18}$ and require extensive chemo-radiation therapy, because of the lack of a proper screening program. ${ }^{19}$

Infection of the cervical epithelium with oncogenic types of HPV is essential to the development of cervical cancer and its precursor lesions. ${ }^{7}$ Western data have documented that HPV DNA was found in $99.7 \%$ of all cervical carcinomas, and cells that derived from those cancers. ${ }^{4}$ Alsobeih et al reported the first prevalence of HPV subtype in cervical cancer patients in the Saudi population. From a total of 100 patients they 


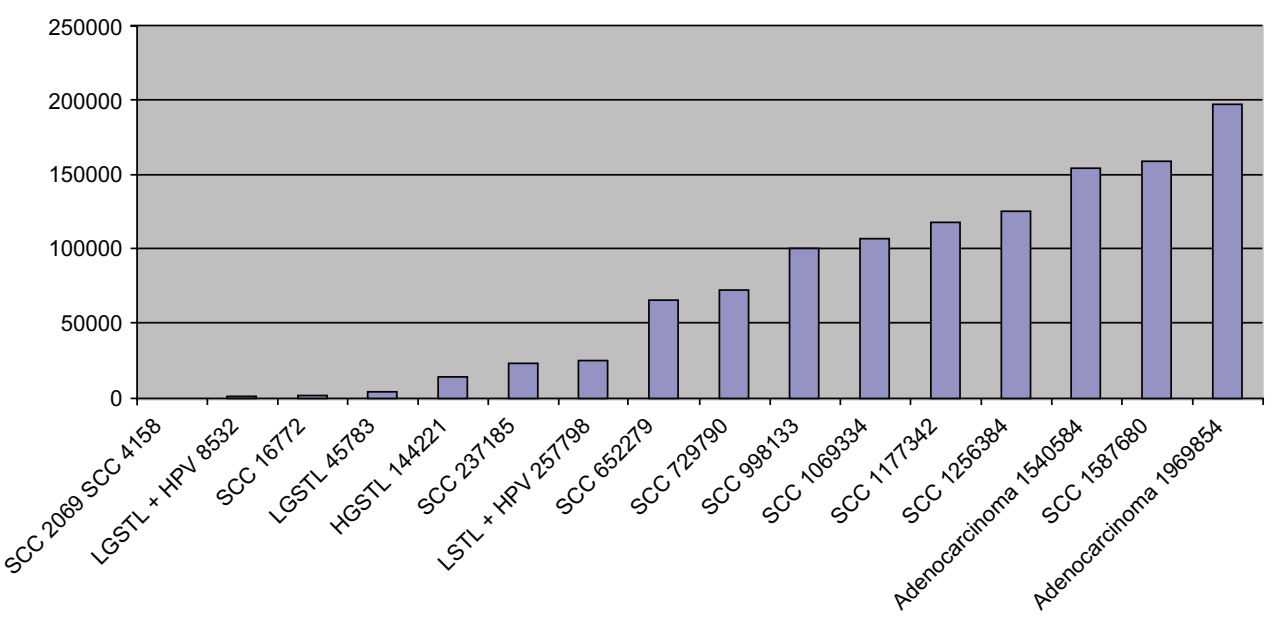

Figure I Relative light unit (RLU) values in positive HPV DNA cervical biopsies with different histopathology diagnosis.

reviewed, they found 89 HPV-positive specimens. Eleven different HPV genotypes were detected, eight high risk $(16,18$, $31,39,45,51,59$, and 73$)$ and three low risk $(6,64$, and 70$)$. Ten patients had double infections involving mainly HPV-16 and 18. The most common genotypes were $16(65.2 \%)$, $31(7.9 \%), 45(6.7 \%), 18(3.4 \%)$, and 73 (2.3\%). However, by considering double infections, HPV-18 became the second most common genotype (10.1\%). The patients' median age was significantly lower $(P=0.028)$ in the HPV-16/18 infected group, compared with other genotypes (44, range $32-76$ vs 49 , range $38-67) .{ }^{20}$ Nevertheless, these results seem to be different from those obtained in another Middle Eastern country where the most common HPV genotype was 33, which was not detected in our patients, followed by 16 and $18 .{ }^{21}$

In a study performed on 120 women attending routine gynecological examination, Al-Muammar et al reported the overall HPV-16/18 prevalence as $31.6 \%$. The prevalences of HPV-16 and -18 alone were $13.3 \%$ and $3.3 \%$, respectively. HPV-16 as a mixed infection with HPV-18 was $15 \%{ }^{22}$

The low prevalence of HPV in the population-based studies of control patients in Spain, was found to be approximately 5.2\%. The Spanish population is considered a lowrisk population due to sexual behaviors and a known low prevalence of $\mathrm{HPV}^{23}$

This study showed that $50 \%$ of patients with high-grade dysplasia were negative for HPV, which is a much higher number than previously published. ${ }^{23-25}$ Of patients with invasive carcinoma, $62 \%$ were positive for HPV; this is a lower number compared with other studies. The typical range is $74 \%-95 \%$ in cervical cancer. ${ }^{20,23-25}$ In addition, $37 \%$ of low grade cases were found to be positive for HPV - a much lower rate than that reported in previously reported studies, which is usually $65-80 \% .{ }^{23-25}$ The low prevalence of HR HPV in our study population may be attributed to the number of biopsies taken from the patients with invasive carcinoma. Multiple biopsies are required to detect HPV DNA by HC2, and also histopathology tests. Only six cases were repeated twice; where only one patient was HPV DNA positive (2.2\%) (RLU $=4158$ ) but HPV DNA negative in another biopsy $(\mathrm{RLU}=118)$. The positive result of this patient correlated with the histopathology result, which was squamous cell carcinoma grade 3 . There was a highly significant difference in RLUs between those women who were reported as having HR HPV infection and those who had negative HPV DNA results. By comparing the RLU values of positive specimens, we found that the signal generated from positive specimens was as high as 1,969,854 RLUs, or as low as 2069 RLUs, as shown in Figure 1. The relative relationships between RLUs and viral load were studied by Schlecht et $\mathrm{al}^{26}$ and Sun et al. ${ }^{27}$ The presence of HR HPV DNA detected by HC2, and a high viral load, were suggested to be strong risk factors for incident-abnormal Pap smear results during monitoring. Hence, assessment of the viral load might be helpful in identifying women at risk for high-grade dysplasia, as reported by Schlecht et al who measured viral burden using PCR, and by Sun et al who measured RLUs using HC2. Schlecht et al concluded that the risk of incident lesions increased with viral load at enrolment and that viral burden appears to have an independent effect on dysplasia incidence. Measurement of viral load, as a surrogate for HPV persistence, may identify women at risk of developing cervical cancer precursors. The recognition of HR HPVs as etiological agents of cervical cancer has increased the demand for testing for HPV, to detect abnormal cervical smears, and for cervical cancer screening, supporting the concept of HPV vaccination against an important subtype. 


\section{Conclusions}

The use of molecular detection of HPV DNA by HC2 in biopsy is easy and effective. Multiple biopsies should be obtained from each patient for testing in order to avoid a false negative result. The present study shows that measuring RLUs by using HC2 is an important indicator in a high viral load in already established invasive disease. This may open the door for more research on this aspect, which may turn out to be a potential prognostic indicator. Although our sample size was very small, our results confirm the finding that HPV contributes to the etiology of cervical cancer in a Muslim society.

The belief that Muslim society has different sexual behavior and cervical cancer etiology from other parts of the word needs to be changed. It is essential to work together to conduct larger local, epidemiological studies in order to determine whether other factors or HPV subtypes may exist in our society. However, acknowledged data on HPV vaccines should not be withheld from those women wishing to consider these data.

\section{Disclosure}

The authors report no conflicts of interest in this work.

\section{References}

1. Parkin DM, Bray F, Ferlay J, Pisani P. Global cancer statistics, 2002. CA Cancer J Clin. 2005;55(2):74-108.

2. Murray CJ, Lopez AD. Mortality by cause for eight regions of the world: global Burden of disease study. Lancet. 1997;349(9061):1269-1276.

3. de Sanjosé S, Diaz M, Castellsagué X, et al. Worldwide prevalence and genotype distribution of cervical human papillomavirus DNA in women with normal cytology: a meta-analysis. Lancet Infect Dis. 2007;7(7):453-459.

4. Bosch FX, Lorincz A, Muñoz N, Meijer CJ, Shah KV. The causal relation between human papillomavirus and cervical cancer. J Clin Pathol. 2002;55(4):244-265.

5. Muñoz N, Bosch FX, de Sanjosé S, et al. The causal link between human papillomavirus and invasive cervical cancer: a populationbased case-control study in Colombia and Spain. Int J Cancer. 1992; 52(5):743-749.

6. Walboomers JM, Jacobs MV, Manos MM, et al. Human papillomavirus is a necessary cause of invasive cervical cancer worldwide. J Pathol. 1999;189(1):12-19.

7. Smith JS, Lindsay L, Hoots B, et al. Human papillomavirus type distribution in invasive cervical cancer and high-grade cervical lesions: a meta-analysis update. Int J Cancer. 2007;121(3):621-632.
8. Ames A, Gravitt P. Human papillomavirus vaccine update. Curr Infect Dis Rep. 2007;9(2):151-158.

9. Helfand RF, Moss WJ, Harpaz R, Scott S, Cutts F. Evaluating the impact of the HIV pandemic on measles control and elimination. Bull World Health Organ. 2005;83(5):329-337.

10. Ghaemmaghami F, Nazari Z, Mehrdad N. Female genital warts. Asian Pac J Cancer Prev. 2007;8(3):339-347.

11. Gazzaz FB. Molecular testing of human papillomavirus in cervical specimens. Saudi Med J. 2007;28(12):1810-1818.

12. Akil N, Yasmeen A, Kassab A, Ghabreau L, Darnel AD, Al Moustafa AE. High-risk human papillomavirus infections in breast cancer in Syrian women and their association with Id-1 expression: a tissue microarray study. Br J Cancer. 2008;99(3):404-407.

13. Altaf FJ. Pattern of cervical smear cytology in western region of Saudi Arabia. Ann Saudi Med. 2001;21(1-2):94-96.

14. Jamal A, Al-Maghrabi JA. Profile of Pap smear cytology in the Western region of Saudi Arabia. Saudi Med J. 2003;24(11):1225-1229.

15. Bhurgri Y, Bhurgri A, Rahim A, et al. The pattern of malignancies in Karachi (1995 to 1996). J Pak Med Assoc. 1999;49(7):157-161.

16. Raza SA, Franceschi S, Pallardy S, et al. Human papillomavirus infection in women with and without cervical cancer in Karachi, Pakistan. Br J Cancer. 2010;102(11):1657-1660.

17. el Dosoky M, Ismail N, Dagastani M. Preinvasive cervical carcinoma in Saudi Arabia. Lancet. 1995;345(8950):650.

18. Manji M. Cervical cancer screening program in Saudi Arabia: action is overdue. Ann Saudi Med. 2000;20(5-6):355-357.

19. Kitchener HC, Symonds P. Detection of cervical intraepithelial neoplasia in developing countries. Lancet. 1999;353(9156):856-857.

20. Alsbeih G, Ahmad R, Al-Harbi N, Aubrey L, Tulbah A, Balaraj K. Prevalence and genotypes' distribution of human papilloma virus in invasive cervical cancer in Saudi Arabia. Gynecol Oncol. 2011;121(3): $522-526$.

21. Darnel AD, Wang D, Ghabreau L, et al. Correlation between the presence of high-risk human papillomaviruses and Id gene expression in Syrian women with cervical cancer. Clin Microbiol Infect. 2010;16(3): 262-266.

22. Al-Muammar T, Al-Ahdal MN, Hassan A, Kessie G, Dela Cruz DM, Mohamed GE. Human papilloma virus-16/18 cervical infection among women attending a family medical clinic in Riyadh. Ann Saudi Med. 2007;27(1):1-5.

23. Muñoz N, Bosch FX, de Sanjosé S, et al. Epidemiologic classification of human papillomavirus types associated with cervical cancer. $N$ Engl J Med. 2003;348(6):518-527.

24. Walboomers JM, Jacobs MV, Manos MM, et al. Human papillomavirus is a necessary cause of invasive cervical cancer worldwide. J Pathol. 1999;189(1):12-19.

25. de Sanjose S, Quint WG, Alemany L, et al. Human papillomavirus genotype attribution in invasive cervical cancer: a retrospective crosssectional worldwide study. Lancet Oncol. 2010;11(11):1048-1056.

26. Schlecht NF, Trevisan A, Duarte-Franco E, et al. Viral load as a predictor of the risk of cervical intraepithelial neoplasia. Int J Cancer. 2003; 103(4):519-524.

27. Sun CA, Liu JF, Wu DM, Nieh S, Yu CP, Chu TY. Viral load of highrisk human papillomavirus in cervical squamous intraepithelial lesions. Int J Gynaecol Obstet. 2002;76(1):41-47.
Pathology and Laboratory Medicine International

\section{Publish your work in this journal}

Pathology and Laboratory Medicine International is a peer-reviewed, open access journal focusing on innovative basic research and translational research related to pathology or human disease. The journal includes original research, updates, case reports, reviews and commentaries on current controversies. The Academic Sponsor

\section{Dovepress}

of this journal is the Chinese American Pathology Association (CAPA). The manuscript management system is completely online and includes a very quick and fair peer-review system. Visit http://www.dovepress.com/testimonials.php to read real quotes from published authors. 